\title{
Optical Methods, Drug Targeting and Skin Aging - An Interesting Mixture of Hot Topics in Skin Pharmacology and Physiology
}

In the present issue of our journal, an interesting mixture of methodical developments for skin pharmacological and physiological measurements and topics related to drug targeting is presented.

The review by Mehling and Fluhr (Skin Pharmacol Physiol 2006;19:182-189) offers an interesting insight into the field of chronobiology. This new field deals with the influence of cyclic time lapses, such as the change between day and night, or the influence of seasonal variations on our organism. The skin, as the largest organ in direct contact with the environment, sensitively reflects this effect.

The penetration of topically applied substances into and through the skin barrier is a regular topic of our journal. Nanoparticles are a promising carrier of drug delivery, as they penetrate efficiently into the hair follicles, if their size is in the same order of magnitude as the surface structure of the hairs. In the paper 'Hair follicles - a long-time reservoir for drug delivery', Lademann et al. (Skin Pharmacol Physiol 2006;19:232-236) demonstrate that nanoparticles are stored up to 10 times longer in the hair follicles than in the stratum corneum.

Luengo et al. (Skin Pharmacol Physiol 2006;19:190-197) investigated the influence of flufenamic acid in poly(lactideco-glycolide) nanoparticles on the transport of flufenamic acid into excised human skin. In contrast to short-time incubation, the nanoencapsulated drug is stored in deeper skin layers, if the incubation time is increased. During the past years, optical and spectroscopic methods have become an efficient tool in noninvasive online analysis of penetration processes and structural changes of tissue. Two-photon laser scanning microscopy was used to analyze the distribution of the nanoencapsulated drug in the skin. The nanoencapsulated drug showed a statistically significant enhanced transport and accumulation. While Luengo et al. investigated the distribution of nanoparticles by 2-photon laser scanning microscopy, Chrit et al. (Skin Pharmacol Physiol 2006;19:207215) used a confocal Raman fiber-optic microprobe for the assessment of a glycerol-based hydration cream in an in vivo randomized study. They demonstrate the potentials of this confocal Raman microprobe in the screening of hydrating agents or molecules under in vivo conditions.

The distinction between intercellular and follicular penetration is still a topic of intensive investigation. Teichmann et al. (Skin Pharmacol Physiol 2006;19:216-223) describe a new method to analyze the different penetration pathways in vivo. They developed a method to selectively block the follicles against the penetration of topically applied substances. By means of this procedure the influence of hair follicles on the penetration and storage of topically applied drugs and cosmetic products can be investigated.

Making a cluster analysis of the dermal permeability of 10 chemicals in 24 chemical mixtures in porcine skin, van der Merwe and Riviere (Skin Pharmacol Physiol 2006;19: 198-206) analyzed whether the efficacy of the penetration of topically applied substances is determined by the pharmacological properties of the agent or by the formulation. These results demonstrate the potential of using cluster analysis of large datasets to identify the effect of consistent solvent and chemical mixtures.

The appearance of the human skin is not only determined by the hydration properties but also by various other factors, such as the concentration of antioxidant substances in the skin. Heinrich et al. (Skin Pharmacol Physiol 2006;19:224231) investigated the influence of 2 different antioxidant supplements composed of carotenoids, vitamin E and selenium on parameters related to skin health and skin aging, whereby the skin density and thickness were determined. A significant increase in both parameters was detectable in the verum groups. The roughness and scaling were improved by a supplementation with antioxidant micronutrients.

Last but not least, we would like to draw your attention to the second meeting of the International Society of Skin Pharmacology and Physiology (ISP), which will be held in Rome from November 5th to 7th, 2006. Additional information is available on the homepage of the ISP (http://www.ispsociety.org/meetings.html).

Jürgen Lademann, Editor Joachim Fluhr, Secretary of ISP

\begin{tabular}{ll}
\hline KARGER & ( 2006 S. Karger AG, Basel \\
Fax +41660-5527/06/0194-0181\$23.50/0 \\
$\begin{array}{l}\text { E-Mail karger@karger.ch } \\
\text { www.karger.com }\end{array}$ & $\begin{array}{l}\text { Accessible online at: } \\
\text { www.karger.com/spp }\end{array}$
\end{tabular}

